Abstract 166 Table 1
\begin{tabular}{|c|c|c|c|}
\hline & $\begin{array}{c}\text { PPCl activation } \\
\text { criteria met }\end{array}$ & $\begin{array}{c}\text { PPCl activation } \\
\text { criteria not met }\end{array}$ & Total \\
\hline $\begin{array}{c}\text { Accepted for PPCl } \\
\text { PPCl }\end{array}$ & True positive 306 & False positive 56 & 362 \\
\hline Total & False negative 15 & True negative 580 & 595 \\
\hline Sensitivity & $95.33 \%$ & $\begin{array}{c}\text { Negative predictive } \\
\text { value }\end{array}$ & 957 \\
\hline Specificity & $91 / 19 \%$ & $\begin{array}{c}\text { Positive predictive } \\
\text { value }\end{array}$ & $84.48 \%$ \\
\hline
\end{tabular}

identified as having been inappropriately turndown on first referral. In 2014, the inappropriate turndown rate had been $3.1 \%$

$322(76.1 \%)$ patients in the turndown population required hospital admission and 290 (48.7\%) were admitted to a Cardiology service. The six-month mortality in the turndown population was $17 \%$ with a cardiac cause of death in $5.7 \%$ of cases. There were $2(13.3 \%)$ deaths in the inappropriate turndown group at six months, both due to cardiac causes.

The sensitivity, specificity positive and negative predictive values are shown in the Table. They indicate a high level of diagnostic accuracy for nurse-delivered ECG interpretation, and show that the system inclines towards greater sensitivity to miss as few patients as possible.

Conclusion In this PPCI service, the majority of patients referred are turned down appropriately. Clinical outcomes were similar among patients in the appropriate and inappropriate groups in the turndown cohort and the mortality rate in this group as a whole is relatively high. The rate of inappropriate turndown has decreased since 2014.

Conflict of Interest Nil

\section{CAN THE USE OF HIGHLY SENSITIVE TROPONIN ASSAY REDUCE THE LENGTH OF HOSPITAL ATTENDANCE AND IMPROVE PATIENT FLOW ON THE ACUTE MEDICAL UNIT?}

'Zoe Stone*, ${ }^{2}$ Elin Powell, ${ }^{3} J o h n$ Hounsell, ${ }^{4}$ Aaron Wong. ${ }^{1}$ Princess of Wales Hospital, Bridgend; ${ }^{2}$ Princess of Wales Hospital, Bridgend; ${ }^{3}$ Princess of Wales Hospital, Bridgend; ${ }^{4}$ Princess of Wales Hospital, Bridgend

\subsection{6/heartjnl-2019-BCS.164}

Introduction The 2015 European Society of Cardiology (ESC) guidelines for investigation and management of non-ST elevation myocardial infarction (NSTEMI) discuss a 'rule in/ rule out' $(0 \mathrm{~h} / \mathrm{h}$ ) algorithm for the use of one-hour highly sensitive troponin assay (figure 1) (1). Following the application of this algorithm to several large cohorts, the negative predictive value for NSTEMI in patients assigned 'rule out' exceeded 98\% according to these guidelines.

Aim The aim of this audit was to evaluate current practice of the assessment of cardiac sounding chest pain on the Acute Medical Unit (AMU) and to establish whether or not the introduction of highly sensitive one-hour troponin assay can shorten length of attendance and improve patient flow through the department.

Method Prospective data collection from October 2018 to December 2018. 99 patients presented to the AMU with cardiac sounding chest pain. Demographic data, ECG findings, troponin results, length of attendances and outcomes (admission, discharge and follow up) were captured.

Results 53\% were male. Mean age was 63.56 patients had initial troponin at presentation only. 12 patients had repeat troponin assays taken following 3 hours; 31 patients had repeat taken after 6 hours. Repeat blood tests for troponin were frequently taken at incorrect times. 9 patients had ischaemic changes on their initial ECG. Acute coronary syndrome was excluded in 88 patients; 26 patients were discharged with Emergency Access Cardiology clinic follow up for further investigation of chest pain. Of those patients who had repeat troponin tests, $77 \%$ of those could potentially have been discharged much sooner (avoiding some overnight stays) if the highly sensitive one-hour troponin assay was available (figure 2).

Conclusion This audit has highlighted several areas of disparity of standard practice in the assessment of chest pain on the AMU. We would like to undertake further work to improve standards. The recommendation of the one-hour troponin protocol suggested by ESC has the potential to significantly 